\title{
An improved ICP algorithm based on Generalized Procrustes Analysis
}

\author{
Wei Wei $\operatorname{Tian}^{1}$ Cheng Jun $\operatorname{Tian}^{1^{*}}{\text { Lei } \operatorname{Han}^{1}}^{1}$ \\ ${ }^{1}$ Dept. of Electronics and Information Engineering, Changchun University of Science and \\ Technolog, China \\ *corresponding author tcjciom@126.com
}

Keywords: point cloud registration; Procrustes analysis; ICP algorithm

Abstract: Point cloud registration often use ICP algorithm to match , which is the classic algorithm of point cloud registration, due to the presence of some defects in ICP algorithm, scholars later have done a lot of improvements. In this paper, we firstly view the traditional ICP algorithms, then we propose the Procrustes analysis into the ICP algorithm for multi view point cloud registration. Simulation results show that this method can resolve the phenomenon in ICP registration algorithm of bifurcation and bloated.

\section{Introduction}

In practical application, we need to scan the object with multi-angle in order to obtain the full 3D point cloud. But different point cloud coordinate system is different. We need to convert multi view point cloud into the same coordinate system. The registration technique is divided into initial registration and accurate registration. The initial registration provides a good initial value for accurate registration. In this paper, we use feature point registration to the initial registration. And accurate registration is based on the ICP algorithm (Iterative Closest Point). ICP algorithm is proposed by Besl and Mackay ${ }^{[1]}$ in 1992. It can solve the problem of free surface matching. It is the basis of accurate registration of point clouds. However, there are some defects, which computing takes lots of time during the registration process,. Many scholars have done a lot of improvements. Zhang $^{[2]}$ uses the tree k-d algorithm to speed up the search of the corresponding point process, set up a dynamic threshold in the iterative process, the matching point to the consistency of the law as a constraint, delete the matching point of the matching points over the dynamic threshold as well as the method to the angle. Dorai $\mathrm{C}^{[3]}$ uses a random sampling method to select the matching points. $\operatorname{Godin}^{[4]}$ proposed that the consistency of the texture and the method of the vertex is used as the constraint to delete invalid corresponding points. Pulli recommends that the percentage of the distance to measure error, and remove the cloud point distance is over $10 \%$.

\section{Initial registration}

There are some initial registration methods, such as the center of the legitimate, Label method, feature extraction. In this paper, we use the label method that is feature point registration, The most common method of this kind of method is the artificial introduction of feature points, such as patch or paste ball, label, according to the feature points in the coordinates of two points are different coordinates system, obtained two point cloud data of the rigid transformation matrix. This method is not very effective in the case of three-dimensional features of objects. The point cloud data from different perspectives can be obtained by using the 3D measurement system. Assumingthat the two set of point cloud data are $A=\left\{a_{i}, i=1,2,3 \ldots k\right\}, B=\left\{b_{i}, i=1,2,3 \ldots k\right\}$, respectively we 
obtain a subset of the point cloud data, $C, D$, where, $c_{i}$ and $d_{i}$ is the one-to-one correspondence. Because of the spatial characteristics of the point cloud data from different angles of view, So the essence of 3D registration is to transform the measured data in different coordinate system, and the problem is transformed into a seeking $\mathrm{R}$ (rotation vector) and $\mathrm{t}$ (translation vector).

The coordinate transformation between 3D data points should be satisfied formula (1).

$$
E=\sum_{i=1}^{n}\left\|c_{i}-\left(R d_{i}+t\right)\right\|^{2}
$$

For the formula (1), we use the SVD matrix decomposition algorithm for solving

\section{Accurate registration}

The preliminary results can be obtained by using feature point registration of initial registration, but it will appear bifurcation phenomenon in the overlapping parts of the two set of point clouds in the result, in order to make the registration error as small as possible between the two points, accurate registration is required after the initial registration is completed.In this paper, the classical Pruk analysis is integrated into the ICP algorithm to achieve accurate registration, it improves both the matching effect and the robustness.

Procrustes analysis is a multivariate statistical method of comparative analysis of shape, it can find the best approximation in the two groups of geometric structures through the rotation, translation, mapping and scaling. That is to say, procrustes analysis selects the appropriate rigid transformation (Separate discussion of scaling) for a structure to estimate the difference between the two groups of structure by fixing a group structure.

The problem of orthogonal Procrustes $(\mathrm{OP})$ is an optimization problem, the orthogonal transformation matrix $\mathrm{R}$ between two $(p \times k)$ dimensional matrix $\mathrm{A}$ and $\mathrm{B}$ is given, then the minimum error can be expressed as

$$
E=A R-B
$$

This method is usually called the extended Procrustes (EOP) which introduce the unknown rotation matrix, the unknown translation vector and the unknown scale factor, at this point the error can be expressed as

$$
E=c A R+j t^{T}-B
$$

Where $\mathrm{j}$ is a unit vector, $\mathrm{c}$ is a scale factor and $\mathrm{t}$ is a two-dimensional translation vector, weighted extended orthogonal Procrustes analysis (weop) is generated by the EOP model with the weight matrix of the component and the points. The minimum error is

$$
\operatorname{tr}\left(\left(c A R+j t^{T}-B\right)^{T} W_{P}\left(c A R+j t^{T}-B\right) W_{K}\right)=e
$$

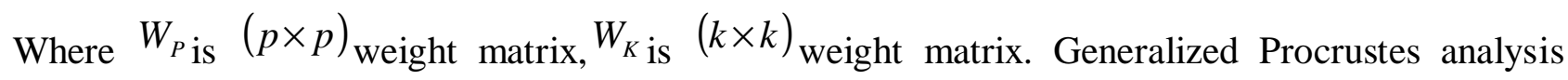
(GPA) is a well known technique, which provides a least squares solution for the existence of the least squares solution when the matrix is over two. It can reduce the least squares objective function.

$$
\operatorname{tr}\left(\sum_{i=1}^{m} \sum_{j=i+1}^{m}\left(\left(c_{i} X_{i} R_{i}+j t_{i}^{T}\right)-\left(c_{j} X_{j} R_{j}+j t_{j}^{T}\right)\right)^{T}\left(\left(c_{i} X_{i} R_{i}+j t_{i}^{T}\right)-\left(c_{j} X_{j} R_{j}+j t_{j}^{T}\right)\right)\right)=e
$$


Where

$\left[X_{1}, X_{2}, \ldots, X_{m}\right]$ are the points in the M model matrix. Another expression of GPA:

$X_{i}^{p}=c_{i} X_{i} R_{i}+j t_{i}^{T}$. The two equations following are equivalent ${ }^{[13]}$.

$$
\begin{array}{r}
\sum_{i<j}^{m}\left\|X_{i}^{p}-X_{j}^{p}\right\|^{2}=\sum_{i<j}^{m} \operatorname{tr}\left(\left(X_{i}^{p}-X_{j}^{p}\right)^{T}\left(X_{i}^{p}-X_{j}^{p}\right)\right) \\
m \sum_{i<j}^{m}\left\|X_{i}^{p}-K\right\|^{2}=m \sum_{i<j}^{m} \operatorname{tr}\left(\left(X_{i}^{p}-K\right)^{T}\left(X_{i}^{p}-K\right)\right)
\end{array}
$$

Where $\mathrm{K}$ is geometrical centroid. Formula (6) determines the number of unknowns $\mathrm{C}, \mathrm{R},{ }^{t}(\mathrm{i}=1, \mathrm{~m})$ in place of the formula (5).Least square estimation of the corresponding centroid of matrix

$$
K=\frac{1}{m} \sum_{i=1}^{m} X_{i}^{p}
$$

Iteration can be found in the solution of system .Initializing centroid $\mathrm{K}$ firstly. The weop is used to solve the transformation parameters of the point matrix of each of the K models in each step. Then, a new centroid can be estimated. The program will continue until the global convergence is obtained by the stability of the centroid K. All of the P points are not always visible in the actual application of the point of the matrix $\left[X_{1}, X_{2}, X_{3} \ldots X_{m}\right]$.In order to solve the problem of missing points, the commander presented a method based on diagonal binary $(\mathrm{P} \times \mathrm{P})$ matrix ${ }_{i}$, where the diagonal elements are 1 or 0 , and the diagonal elements are 1 or 0 ,this solution is considered to be the zero weight of the missing point. The least squares objective function when losing point is defined as follows:

$$
m \sum_{i<j}^{m}\left\|X_{i}^{p}-K\right\|^{2}=m \sum_{i<j}^{m} \operatorname{tr}\left(\left(X_{i}^{p}-K\right)^{T} M_{i}\left(X_{i}^{p}-K\right)\right)
$$

Where

$$
K=\left(\sum_{i=1}^{m} M_{i}\right)^{-1}\left(\sum_{i=1}^{m} M_{i}\left(c_{i} X_{i} T_{i}+j t_{i}^{T}\right)\right)
$$

In order to obtain a more general scheme, the combined weighted and the missing points solution should be considered. A product matrix is obtained by multiplying the weight matrix $P_{i}$ by the binary matrix $_{i}, D_{i}=P_{i} M_{i}$. The corresponding least squares objective function are the following:

$$
m \sum_{i<j}^{m}\left\|X_{i}^{p}-K\right\|^{2}=m \sum_{i<j}^{m} \operatorname{tr}\left(\left(X_{i}^{p}-K\right)^{T} P_{i} M_{i}\left(X_{i}^{p}-K\right)\right)
$$

Where

$$
K=\left(\sum_{i=1}^{m} P_{i} M_{i}\right)^{-1}\left(\sum_{i=1}^{m} P_{i} M_{i}\left(c_{i} X_{i} T_{i}+j t_{i}^{T}\right)\right)
$$

Procrustes Analysis Generalized (GPA) is used in this paper to analyze a large number of samples, which is used to arrange the data and eliminate the irrelevant components. We can directly compare the removal of the other point cloud matrix data with the original point cloud matrix by GPA, in 
order to determine the importance of the point cloud to maintain the origin of the cloud matrix.

In this paper, the generalized Procrustes analysis for the following specific algorithm to solve the point cloud registration:

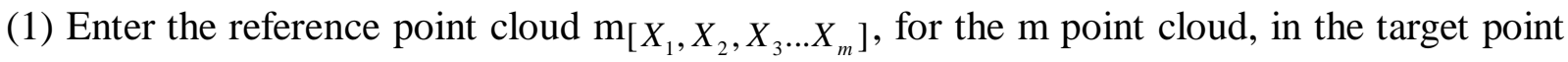
cloud using the Euclidean distance to find the nearest point, to use k-d tree can improve the search speed;

(2) In finding the nearest point pair, the set of equations (12) defines a new K, RST, for the $\mathrm{m}$ point cloud; $\left[T_{1}, T_{2}, T_{3} \ldots T_{m}\right]$ is the RST parameter of the $\mathrm{m}$ point cloud;

(3) Using the formula (11) to estimate the parameters of the RST transformation, the optimal translation vector and rotation matrix are obtained;

(4) Target point cloud using the estimated parameters. The translation and rotation of the translated vector and rotation matrix are converted into the reference frame of the model point cloud, which can make the target data point cloud unified to the reference frame of the model point cloud;

(5) According to the formula (4) to determine whether the error is convergence, determine whether or not to be equal to I (I as set value), whether to continue iterative.

\section{Simulation results}

The experiment is carried out on MatlabR2014a with Bunny point cloud data, Figure a is the result of the traditional ICP algorithm registration, it is very obvious that there is the bifurcation phenomenon in the rabbit's ear part of the bifurcation, and figure B is the result of the GPA-ICP algorithm, a good solution to the bifurcation phenomenon, part of the rabbit ears very clear.

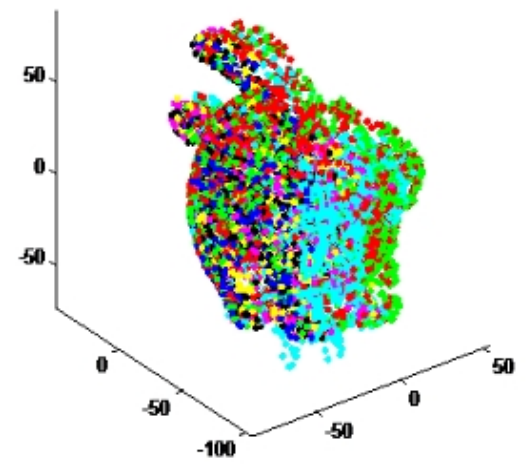

Figure a

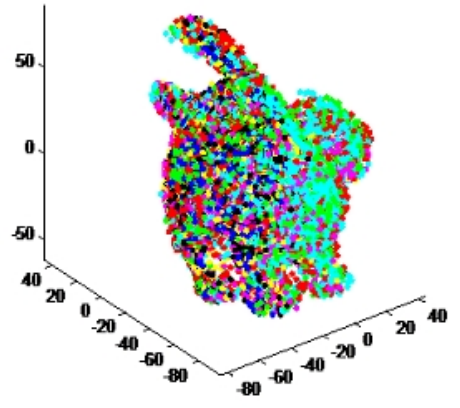

Figure $b$

\section{Summary}

The initial registration using feature point matching algorithm, accurate registration using GPA- ICP algorithm, the ICP algorithm was improved effectively for unknown 3D point cloud registration, the registration speed is improved. Experiments show that the GPA-ICP algorithm has better effect in the multi view point cloud registration, and it can solve some defects of the traditional ICP algorithm, so that the bifurcation phenomenon disappears and a good registration result is obtained.

\section{References}

[1] Paul J. Besl, Neil D. McKay. A method for registration of 3-D shapes [J]. IEEE Transactions on Pattern Analysis and Machine Intelligence, 1992. 14(2): 239-256. 
[2] Zhang Z. Iterative point matching for registration of free-form curves and surfaces. International journal of computer vision, 1994, 13(2): 119 152.

[3] Dorai C, Wang G, Jain A K. Registration and integration of multiple object views for 3D model construction. Pattern Analysis and Machine Intelligence, IEEE Transactions on, 1998, 20(1): 83 89.

[4] Godin G Rioux M, Baribeau R. Three-dimensional registration using range and intensity information. Photonics for Industrial Applications International Society for Optics and Photonics, 1994: 279 290. 